\title{
Particle Motion over the Surface of a Cylinder, which Performs Translational Oscillations in a Vertical Plane
}

\author{
Serhii Pylypaka ${ }^{1, a}$, Mykola Klendii ${ }^{2, b}$, Tetyana Kremets ${ }^{1}$, and Oleksandra Klendii ${ }^{2, c^{*}}$ \\ 1 National University of Life and Environmental Sciences of Ukraine, Heroiv Oborony Str., 15, Kyiv, \\ Ukraine; \\ 2 Separated Subdivision of National University of Life and Environmental Sciences of Ukraine Berezhany \\ Agrotechnical Institute, Akademichna Str., 20, Berezhany, Ukraine \\ E-mail: apsf55@ukr.net, bklendiy@ukr.net, cklendii_o@ukr.net (Corresponding author)
}

\begin{abstract}
Generalized differential equations of relative particle motion over the internal surface of an inclined cylinder, which performs an oscillating motion, have been formulated. All the points of a cylinder trace ellipses in vertical planes. Such cases of cylinder oscillation, when either semi-axes or one of them is equal to zero, that is to say, a cylinder performs reciprocating motion, have been considered. The equations have been solved applying numerical methods and trajectories of relative particle motion over the surface of a cylinder have been built. Graphs of other kinematic characteristics as a timevarying function have been presented. Certain cases, when a cylinder axis is located horizontally or at an angle to a horizontal plane, have been considered.
\end{abstract}

Keywords: inclined cylinder, oscillating motion, vertical plane, particle, differential equations, kinematic parameters.

ENGINEERING JOURNAL Volume 22 Issue 3

Received 9 September 2017

Accepted 14 March 2018

Published 28 June 2018

Online at http://www.engj.org/

DOI:10.4186/ej.2018.22.3.83 


\section{Introduction}

An inclined plane is a universal structural element of numerous agricultural machines [1]. In course of processing technological material moves over it. The most investigated is particle motion over a horizontal plane, which performs oscillating rectilinear or circulatory motion. In case of an inclined plane, investigations are usually conducted at its rectilinear reciprocating oscillations in horizontal direction, in the direction of plane inclination or in transverse direction [1]. If there are curvilinear plane oscillations, when all its points trace ellipses and the plane itself has an inclination, the movement of technological material changes significantly. If a cylinder is taken instead of a plane and it performs the same oscillations, the solutions to the problem for investigating relative particle motion over its surface change significantly.

Except for the fundamental monograph [1], which considers rectilinear reciprocating oscillations, there are papers devoted to curvilinear plane oscillations. Generally, the problem of material particle motion over the plane, which performs circular oscillating motion, has been solved by M. Ye. Zhukovskyi in geometrical interpretation [2] and has been generalized and applied to the cases of elliptical vibrations by I.I. Blekhman $[3,4,10]$. P.M. Vasylenko has set up differential equations of particle motion in projections onto a moving system of axes, which is rigidly connected with an oscillating plane [1] and I.I. Blekhman - in projections onto the axes of a fixed coordinate system. P.M. Zaika has considered particle motion over operating surfaces of vibrating grain-cleaning machines [5,9]. Material particle motion over a rough surface of a cylinder, all the points of which trace circles in horizontal planes, has been investigated in paper [6]. Relative particle motion over the surface of a helicoid, which rotates about its vertical axis, has been considered in paper [7].

\section{Material and Method}

Let us locate the lower half of a cylinder in such a way that its axis is inclined to a horizontal plane at an angle $\beta$ (Fig. 1). First of all, let us write the equation of a cylinder with a horizontal axis [8]:

$$
X=u ; \quad Y=R \cos \alpha ; \quad Z=-R \sin \alpha,
$$

where $R$ - radius of a cylinder; $\alpha, u$ - independent variables of a surface, $a$ - angle of rotation of a cylinder point about its axis; $u$-length of rectilinear generator of a cylinder.

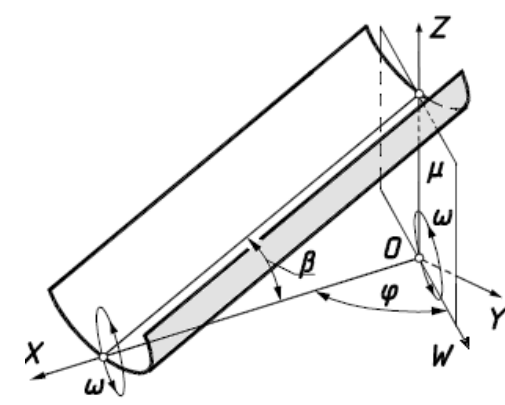

(a)

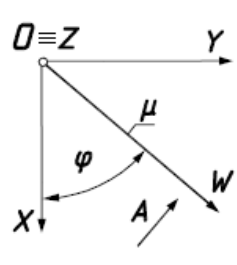

(b)

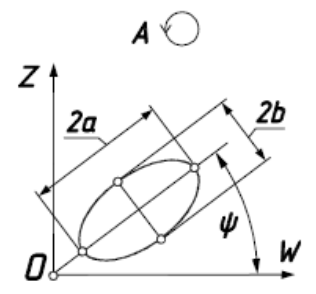

(c)

Fig. 1. Graphic illustrations of an inclined cylinder location and the direction of its oscillations:

(a) Location of a cylinder in $O X Y Z$ coordinates;

(b) Location of a vertical plane $\mu$ in $O X Y Z$ coordinates;

(c) Location of an ellipse in a plane $\mu$ with $O Z W$ axes.

Let us turn the cylinder (1) through an angle $\beta$ about $O Y$ axis. A parametric equation of the turned cylinder can be written as:

$$
X=u \cos \beta+R \sin \beta \sin \alpha ; \quad Y=R \cos \alpha ; \quad Z=u \sin \beta-R \cos \beta \sin \alpha .
$$

A cylinder performs translational oscillations in such a way that all its points generally trace ellipses in vertical parallel planes. Figure 1(a) shows a general case, when a cylinder is rigidly attached to OXYZ coordinate system, all the points of which, together with a cylinder, move in ellipses that are placed in 
vertical planes, which are parallel to plane $\mu$. Plane $\mu$ with $O Z W$ axes (Figs. 1(a)-(b)), where there are ellipses, makes an angle $\varphi$ with $O X Z$ coordinate plane. This angle can be equal to the value ranging from $0^{\circ}$ to $90^{\circ}$. If $\varphi=0^{\circ}$, there are longitudinal oscillations, if $\varphi=90^{\circ}$, there are transverse oscillations. If there is the case presented in Fig. 1(a), this angle is equal to $90^{\circ}$. In a plane $\mu$ let us plot an ellipse with a major semi-axis $a$ and a minor semi-axis $b-a$ trajectory of translational oscillations of all the points of a cylinder (Fig. 1(c)). The direction of the major axis of an ellipse is set up by the angle $\psi$ of its inclination to a horizontal plane. If $b=0$, a cylinder performs reciprocating oscillations along the preset direction of the major axis, if $a=b$, the trajectory of oscillatory motion is in the form of circles (here, the value of angle $\psi$ does not make any difference). We consider absolute particle motion in projections onto the axes of OXYZ coordinate system. Absolute trajectory of a particle can be written as a sum of translational motion of a cylinder, all the points of which in a general case trace ellipses, and relative motion of a particle over the surface of a cylinder:

$$
x=x_{n}+x_{v} ; \quad y=y_{n}+y_{v} ; \quad z=z_{n}+z_{v},
$$

where $x_{n}=x_{n}(t) ; y_{n}=y_{n}(t) ; z_{n}=z_{n}(t)$ - trajectory of relative motion of a cylinder as a time function $t$, $x_{v}=x_{v}(t) ; y_{v}=y_{v}(t) ; z_{v}=q_{v}(t)$ - trajectory of relative motion of a particle over the surface of a cylinder as a time function $t$.

Every point of a cylinder traces an ellipse with semi-axis $a$ and $b$. A radius vector of an ellipse point in the plane $\mu$ of its location has the following coordinates: $\{a \cos \omega t ; b \sin \omega t\}$, where $\omega$ - frequency of oscillations (if $a=b$ - angular velocity of rotation of cylinder points around circles), $t$ - time, independent variable. The axes of such an ellipse are parallel to the axes $O X$ and $O W$. Applying the known rotation formula, let us rotate it through an angle $\psi$. Afterwards, a radius vector of an ellipse point can be written as:

$$
\{-a \cos \psi \cos \omega t+b \sin \psi \sin \omega t ; \quad a \sin \psi \cos \omega t+b \cos \psi \sin \omega t\}
$$

Taking into consideration the angle $\varphi$ between the planes $O Z X$ and $O Z W$, a parametric equation of an ellipse in projections onto the axes of $O X Y Z$ coordinate system can be written as:

$$
\begin{array}{ll}
x_{n}=(a \cos \psi \cos \omega t+b \sin \psi \sin \omega t) \cos \varphi ; & x_{n}=(a \cos \psi \cos \omega t+b \sin \psi \sin \omega t) \cos \varphi ; \\
y_{n}=(a \cos \psi \cos \omega t+b \sin \psi \sin \omega t) \sin \varphi ; & y_{n}=(a \cos \psi \cos \omega t+b \sin \psi \sin \omega t) \sin \varphi ; \\
z_{n}=-a \sin \psi \cos \omega t+b \cos \psi \sin \omega t . & z_{n}=-a \sin \psi \cos \omega t+b \cos \psi \sin \omega t .
\end{array}
$$

A particle slides over a cylinder along a certain trajectory. We obtain the equation of a trajectory, if we correlate independent variables $a$ and $u$ of the surface (2). This correlation can be written by means of time $t$, that is to say, the coordinates of a particle on the surface of a cylinder are time functions: $a=a(t)$ and $u=u(t)$. In this case, relative particle motion (trajectory on a cylinder) can be described by the following equations:

$$
\begin{aligned}
& x_{v}=u \cos \beta+R \sin \beta \sin \alpha ; \\
& y_{v}=R \cos \alpha ; \\
& z_{v}=u \sin \beta-R \cos \beta \sin \alpha .
\end{aligned}
$$

By summing translational (5) and relative (6) motions applying formula (3), we obtain an equation of absolute trajectory of a particle:

$$
\begin{aligned}
& x=(a \cos \psi \cos \omega t+b \sin \psi \sin \omega t) \cos \varphi+u \cos \beta+R \sin \beta \sin \alpha ; \\
& y=(a \cos \psi \cos \omega t+b \sin \psi \sin \omega t) \sin \varphi+R \cos \alpha ; \\
& z=-a \sin \psi \cos \omega t+b \cos \psi \sin \omega t+u \sin \beta-R \cos \beta \sin \alpha .
\end{aligned}
$$

By setting up the values of semi-axis $a$ and $b$, we can obtain different trajectories of relative motion of a cylinder (ellipses, circles, rectilinear segments), which can be oriented in vertical planes in all possible positions by means of respective values of angles $\varphi$ and $\psi$.

The dependences $a=a(t)$ and $u=u(t)$, which describe trajectory of relative motion (sliding of a particle over the surface of a cylinder), are the unknown functions that must be found. After differentiating Eq. (6) according to time $t$, we find projections of absolute velocity of a particle: 


$$
\begin{aligned}
& x^{\prime}=\omega(-a \cos \psi \sin \omega t+b \sin \psi \cos \omega t) \cos \varphi+u^{\prime} \cos \beta+R \alpha^{\prime} \sin \beta \sin \alpha \\
& y^{\prime}=\omega(-a \cos \psi \sin \omega t+b \sin \psi \cos \omega t) \sin \varphi-R \alpha^{\prime} \cos \alpha \\
& z^{\prime}=\omega(a \sin \psi \sin \omega t+b \cos \psi \cos \omega t)+u^{\prime} \sin \beta-R \alpha^{\prime} \cos \beta \cos \alpha .
\end{aligned}
$$

Differentiation of expressions (8) provides projections of absolute acceleration:

$$
\begin{aligned}
& x^{\prime \prime}=-\omega^{2}(a \cos \psi \cos \omega t+b \sin \psi \sin \omega t) \cos \phi+u^{\prime \prime} \cos \beta+R \sin \beta\left(\alpha^{\prime \prime} \cos \alpha-\alpha^{\prime 2} \sin \alpha\right) ; \\
& y^{\prime \prime}=-\omega^{2}(a \cos \psi \cos \omega t+b \sin \psi \sin \omega t) \sin \phi-R\left(\alpha^{\prime \prime} \sin \alpha+\alpha^{\prime 2} \cos \alpha\right) ; \\
& z^{\prime \prime}=\omega^{2}(a \sin \psi \cos \omega t-b \cos \psi \sin \omega t)+u^{\prime \prime} \sin \beta-R \cos \beta\left(\alpha^{\prime \prime} \cos \alpha-\alpha^{\prime 2} \sin \alpha\right) .
\end{aligned}
$$

Let us set up motion equation in the form of $m \bar{w}=\bar{F}$, where $m$ - mass of a particle, $\bar{w}-$ vector of absolute acceleration, $\bar{F}$ - resultant vector of the forces exerted upon a particle. Such forces are weight force $m g\left(g=9,81 \mathrm{~m} / \mathrm{s}^{2}\right)$, reaction $N$ of the surface of a cylinder and friction force $f N$ when particle is sliding over the surface of a cylinder $(f$ - friction coefficient). All the forces must be projected onto the axes of $O X Y Z$ coordinate system.

Weight force is down-directed, thus, its projections can be written as:

$$
\{0 ; 0 ;-m g\}
$$

Reaction $N$ of the surface of a cylinder is directed normally to it and is determined by the vector product of two vectors, which are tangent to the coordinate lines of a cylinder. The projections of these vectors are partial derivatives of Eq. (2):

$$
\begin{aligned}
\frac{\partial X}{\partial \alpha} & =R \sin \beta \cos \alpha ; & \frac{\partial Y}{\partial \alpha}=-R \sin \alpha ; & \frac{\partial Z}{\partial \alpha}=-R \cos \beta \cos \alpha ; \\
\frac{\partial X}{\partial u} & =0 ; & \frac{\partial Y}{\partial u}=\cos \beta \sin \alpha ; & \frac{\partial Z}{\partial u}=\sin \beta
\end{aligned}
$$

After vector multiplication of vectors (11) and after reducing of the obtained vector to a unit one, projections of a normal vector to the surface can be written as:

$$
\{-\sin \beta \sin \alpha ; \quad-\cos \alpha ; \quad \cos \beta \sin \alpha\}
$$

Since friction force is directed at a tangent to the trajectory of relative motion of a particle to the opposite side, let us find projections of a tangent vector. They are determined by the first derivatives of Eq. (6):

$$
x_{v}^{\prime}=u^{\prime} \cos \beta+R \alpha^{\prime} \sin \beta \cos \alpha ; \quad y_{v}^{\prime}=-R \alpha^{\prime} \sin \alpha ; \quad z_{v}^{\prime}=u^{\prime} \sin \beta-R \alpha^{\prime} \cos \beta \cos \alpha .
$$

Geometric sum of the components (13) yields the velocity of particle sliding over the surface of a cylinder in relative motion:

$$
V_{v}=\sqrt{x_{v}^{\prime 2}+y_{v}^{\prime 2}+z_{v}^{\prime 2}}=\sqrt{u^{\prime 2}+R^{2} \alpha^{\prime 2}}
$$

We obtain a unit tangent vector in projections onto the axes of $O X Y Z$ coordinate system from dividing projections (13) by the value of vector (14):

$$
\left\{\frac{u^{\prime} \cos \beta+R \alpha^{\prime} \sin \beta \cos \alpha}{\sqrt{u^{\prime 2}+R^{2} \alpha^{\prime 2}}} ; \quad-\frac{R \alpha^{\prime} \sin \alpha}{\sqrt{u^{\prime 2}+R^{2} \alpha^{\prime 2}}} ; \quad \frac{u^{\prime} \sin \beta-R \alpha^{\prime} \cos \beta \cos \alpha}{\sqrt{u^{\prime 2}+R^{2} \alpha^{\prime 2}}}\right\}
$$


Let us break down vector equation $m \bar{w}=\bar{F}$ in projections onto coordinate axes, taking into consideration that friction force $f N$ is directed along a unit vector (15) to the side opposite to it:

$$
\begin{aligned}
& m x^{\prime \prime}=-N \sin \beta \sin \alpha-f N \frac{u^{\prime} \cos \beta+R \alpha^{\prime} \sin \beta \cos \alpha}{\sqrt{u^{\prime 2}+R^{2} \alpha^{\prime 2}}} \\
& m y^{\prime \prime}=-N \cos \alpha+f N \frac{R \alpha^{\prime} \sin \alpha}{\sqrt{u^{\prime 2}+R^{2} \alpha^{\prime 2}}} ; \\
& m z^{\prime \prime}=-m g+N \cos \beta \sin \alpha-f N \frac{u^{\prime} \sin \beta-R \alpha^{\prime} \cos \beta \cos \alpha}{\sqrt{u^{\prime 2}+R^{2} \alpha^{\prime 2}}} .
\end{aligned}
$$

Let us substitute other derivatives (projections of absolute acceleration) from (9) into Eq. (16) and we get the system of three equations:

$$
\begin{aligned}
& m\left[-\omega^{2}(a \cos \psi \cos \omega t+b \sin \psi \sin \omega t) \cos \phi+u^{\prime \prime} \cos \beta+R \sin \beta\left(\alpha^{\prime \prime} \cos \alpha-\alpha^{\prime 2} \sin \alpha\right)\right]= \\
& =-N \sin \beta \sin \alpha-f N \frac{u^{\prime} \cos \beta+R \alpha^{\prime} \sin \beta \cos \alpha}{\sqrt{u^{\prime 2}+R^{2} \alpha^{\prime 2}}} \\
& m\left[-\omega^{2}(a \cos \psi \cos \omega t+b \sin \psi \sin \omega t) \sin \phi-R\left(\alpha^{\prime \prime} \sin \alpha+\alpha^{\prime 2} \cos \alpha\right)\right]=-N \cos \alpha+f N \frac{R \alpha^{\prime} \sin \alpha}{\sqrt{u^{\prime 2}+R^{2} \alpha^{\prime 2}}} \\
& m\left[\omega^{2}(a \sin \psi \cos \omega t-b \cos \psi \sin \omega t)+u^{\prime \prime} \sin \beta-R \cos \beta\left(\alpha^{\prime \prime} \cos \alpha-\alpha^{\prime 2} \sin \alpha\right)\right]= \\
& =-m g+N \cos \beta \sin \alpha-f N \frac{u^{\prime} \sin \beta-R \alpha^{\prime} \cos \beta \cos \alpha}{\sqrt{u^{\prime 2}+R^{2} \alpha^{\prime 2}}}
\end{aligned}
$$

System (17) includes three unknown functions: $N=N(t), u=u(t)$ and $a=a(t)$. Having solved it for $N, u^{\prime \prime}$ and $\alpha^{\prime \prime}$, we obtain the following expressions:

$$
\begin{aligned}
& \alpha^{\prime \prime}=-f \frac{\alpha^{\prime 3}}{\sqrt{u^{\prime 2}+R^{2} \alpha^{\prime 2}}} R-K \omega^{2}\left(\frac{\sin \alpha}{R}+f \frac{\alpha^{\prime} \cos \alpha}{\sqrt{u^{\prime 2}+R^{2} \alpha^{\prime 2}}}\right) \sin \varphi+ \\
& +\left(\frac{\cos \alpha}{R}-f \frac{\alpha^{\prime} \sin \alpha}{\sqrt{u^{\prime 2}+R^{2} \alpha^{\prime 2}}}\right)\left(L \omega^{2} \sin \beta \cos \varphi+M \cos \beta\right) ; \\
& u^{\prime \prime}=-g \sin \beta+A b \omega^{2} \sin \omega t+B a \omega^{2} \cos \omega t-f \frac{u^{\prime} P}{\sqrt{u^{\prime 2}+R^{2} \alpha^{\prime 2}}} ; \\
& N=m P .
\end{aligned}
$$

System (18) is quite lengthy in spite of the fact that we have substituted some of the expressions for invariables and variables with symbols. Such invariables are the following ones:

$$
A=\cos \beta \sin \psi \cos \varphi+\sin \beta \cos \psi ; \quad B=\cos \beta \cos \psi \cos \varphi-\sin \beta \sin \psi .
$$

The following symbols replace the variables:

$$
\begin{aligned}
& K=\sin \beta \cos \varphi \sin \alpha+\sin \varphi \cos \alpha ; \quad L=a \cos \psi \cos \omega t+b \sin \psi \sin \omega t ; \\
& M=g+\omega^{2}(a \sin \psi \cos \omega t-b \cos \psi \sin \omega t) ; \quad P=R \alpha^{\prime 2}+K L \omega^{2}+M \cos \beta \sin \alpha .
\end{aligned}
$$

Solution of Eq. (17) for $N, u^{\prime \prime}$ and $a^{\prime \prime}$, as well as numerical integration of the system of differential Eq. (18) for finding initial functions, is possible due to modern software. Let us consider certain cases.

A fixed cylinder $(\omega=0)$. In this case the value of angles $\varphi$ and $\psi$ does not make a difference. Let us consider the case, when the generators of a cylinder are inclined at a friction angle to a horizontal plane, that is to say, $\beta=\operatorname{arctg} f$. The system of differential Eq. (18) and the expression for surface reaction $N$ take a simplified form: 


$$
\begin{aligned}
& \alpha^{\prime \prime}=\frac{g}{R} \cos \alpha \cos \beta-\frac{\alpha^{\prime}}{\sqrt{u^{\prime 2}+R^{2} \alpha^{\prime 2}}}\left(R \alpha^{\prime 2} \operatorname{tg} \beta+g \sin \alpha \sin \beta\right) ; \\
& u^{\prime \prime}=-g \sin \beta-\frac{u^{\prime}}{\sqrt{u^{\prime 2}+R^{2} \alpha^{\prime 2}}}\left(R \alpha^{\prime 2} \operatorname{tg} \beta+g \sin \alpha \sin \beta\right) ; \\
& N=m\left(R \alpha^{\prime 2}+g \cos \beta \sin \alpha\right) .
\end{aligned}
$$

For a separate case, when an initial value of angle $a$ equals to $90^{\circ}$, that is to say, a particle is located on a lower generator of a cylinder and $a^{\prime}=0$, that is to say, there is no initial angular velocity of a particle in the direction opposite to the generators of a cylinder, the first equation of the system is changed into an identical equation, that is to say, the left and the right members are equal to zero. In the second equation the right member also changes into zero on condition that there is a negative sign before the root. Thus, $u^{\prime}=$ const, that is to say, the speed of particle movement along a lower generator is stable, which is to be expected. Surface reaction is stable as well: $N=m g \cos \beta$. The same result is obtained from numerical integration of Eq. (19) under the initial conditions $\alpha=\pi / 2$ and $a^{\prime}=0$.

\section{Result}

The case, when $a=0, a^{\prime}=0$ and $u^{\prime}=0$, that is to say, initial velocity of a particle equals to zero and it begins its motion from the side generator at the level of a cylinder axis, has been considered. In such a case its motion is oscillatory, which is stabilized afterwards and the trajectory approaches to a lower generator (Fig. 2(a)). There are graphic illustrations for friction coefficient $f=0.3$, that is to say, $\beta=16,7^{0}, R=0.2 \mathrm{~m}$, time of movement $t=2.5 \mathrm{~s}$.

In both cases velocity $V$ becomes stabilized afterwards and the trajectory of motion approaches a lower generator of a cylinder, here, after stabilization of motion, velocity is greater in the second case. Surface reaction becomes stable afterwards as well (the graph represents its change for a particle mass $m=0.01 \mathrm{~kg}$ ).

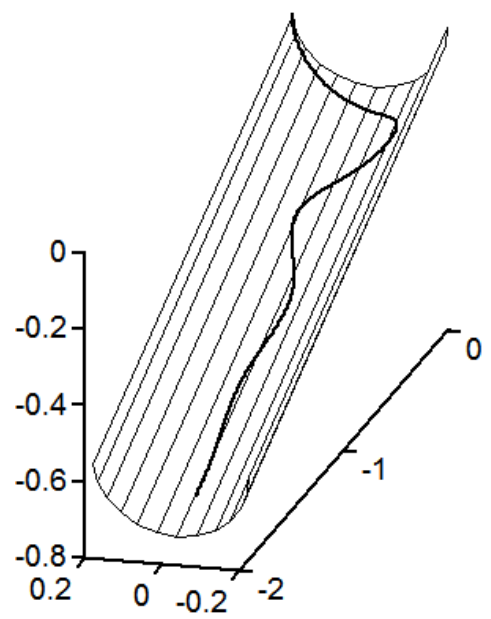

(a)

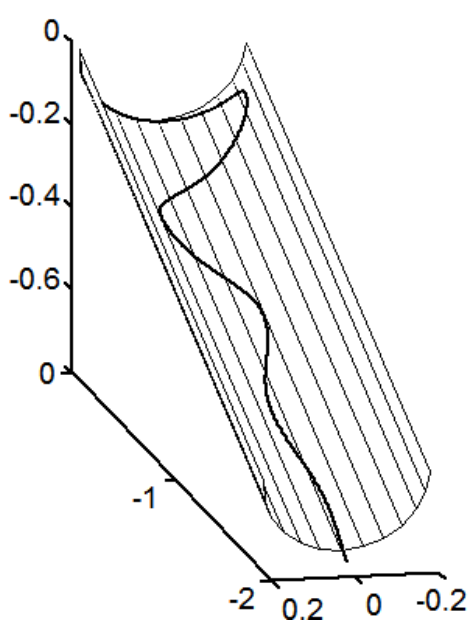

(b)
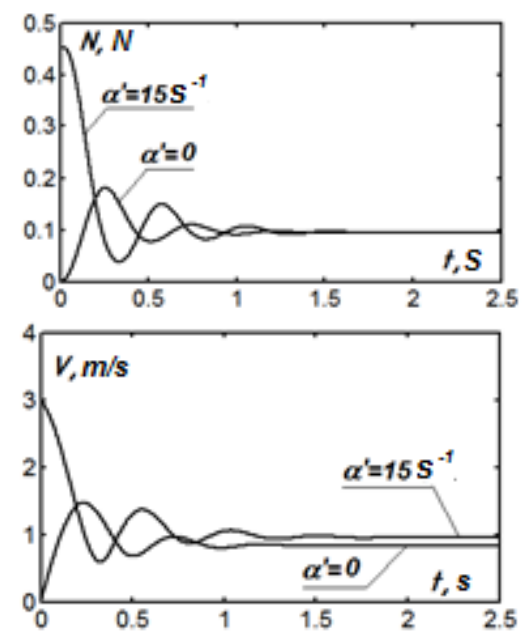

(c)

Fig. 2. Graphic illustrations of particle motion over a fixed cylinder, which is inclined at a friction angle $(\beta=\operatorname{arctg} f)$ :

(a) Trajectory of a particle, which begins its motion from the state of rest;

(b) Trajectory of a particle with initial angular velocity $a^{\prime}=15 s^{-1}$;

(c) Curves of surface reaction $N$ and velocity $V$ of particle motion.

A cylinder performs rectilinear reciprocating oscillations in a horizontal plane $(b=0, \psi=0)$. At such oscillation the height of cylinder points does not change. First of all, let us consider relative particle motion over a horizontal cylinder $(\beta=0)$, which performs oscillations in transverse direction $\left(\varphi=90^{0}\right)$. Obviously, the trajectory of particle sliding is a circular arc of the cross-section of a cylinder, where it oscillates changing the direction of its motion. Such oscillation is possible on condition that at the beginning of its motion 
relative velocity is equal to zero. We set up initial velocity $u^{\prime}=2 \mathrm{~m} / \mathrm{s}$ along the axis of a cylinder. Applying numerical integration of system (18) at $R=0.2 \mathrm{~m}, a=0.1 \mathrm{~m}, a=\pi / 2, a^{\prime}=0, f=0.3$ and various values of $\omega$, we obtain relative trajectory of motion, which is represented in Fig. 3(a). A double-headed arrow line shows the direction of reciprocating oscillations. A particle begins its sliding from a lower generator of a cylinder and in a certain time period its motion is stabilized, afterwards it begins to slide around a circle. With an increase in the frequency of oscillations, a particle covers a greater distance along a cylinder axis within the same time period $(t=2.5 \mathrm{~s})$. This is also represented in Fig. 3(c), which shows a distance $u$ curve along the axis of a cylinder. Figure $3(\mathrm{~b})$ represents the trajectory of relative particle motion under previous initial conditions, except for the angle $\varphi$, which is equal to $45^{0}$ in this case.

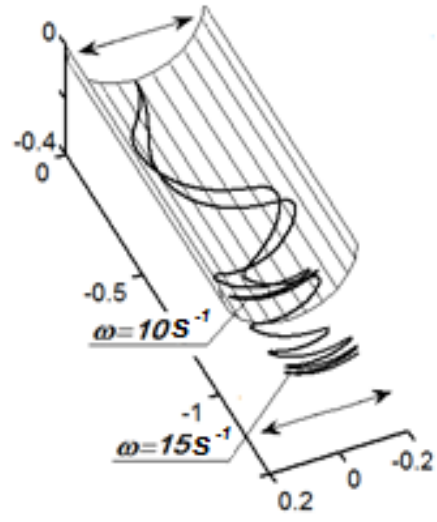

a)

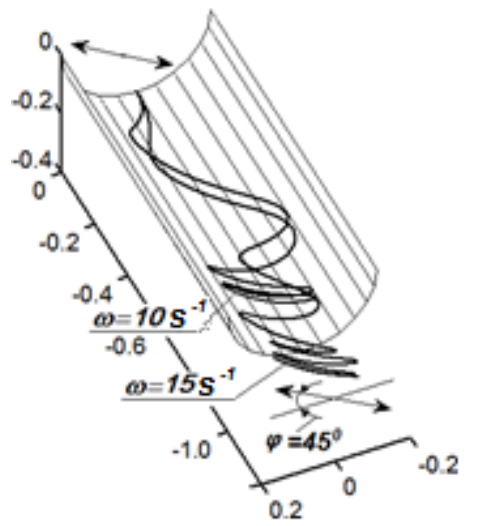

b)

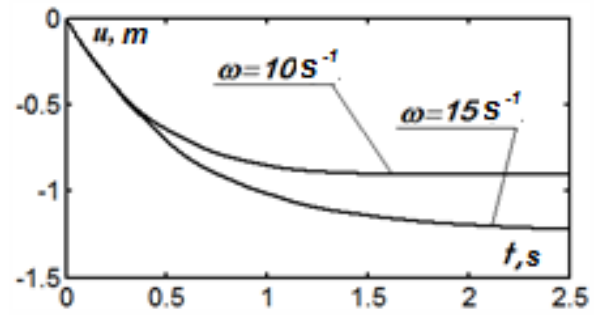

c)

Fig. 3. Graphic illustrations of particle motion over a horizontal cylider, which performs rectilinear recipricating oscillations in a horizontal plane at initial velocity $u^{\prime}=2 \mathrm{~m} / \mathrm{s}$ along its axis:

(a) Trajectory of a particle at $\varphi=90^{\circ}$;

(b) Trajectory of a particle at $\varphi=45^{\circ}$;

(c) Curves of the distance $u$ that a particle covers along a cylinder axis.

Let us consider relative particle motion at transverse rectilinear oscillations of a cylinder at the preset angle $\beta$ of its inclination to a horizontal plane. As it is known, a particle cannot begin its motion over a fixed plane from the state of rest, if its inclination angle is less than a friction angle. The same is related to a cylinder, if a particle is located on its lower generator (that is to say, at $a=\pi / 2$ ). However, the situation changes at oscillatory motion of a cylinder. Even at insignificant inclination angles of an oscillating cylinder, a particle begins to slide over its surface. Figure 4 shows relative trajectories of particle sliding, plotted at $\beta=2^{0}, \mathrm{R}=0.2 \mathrm{~m}, a=0.1 \mathrm{~m}, a=\pi / 2, a^{\prime}=0, u^{\prime}=0, f=0.3$.

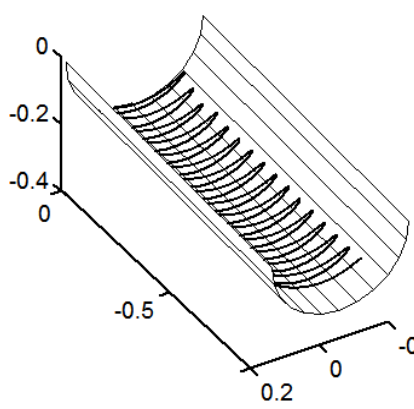

(a)

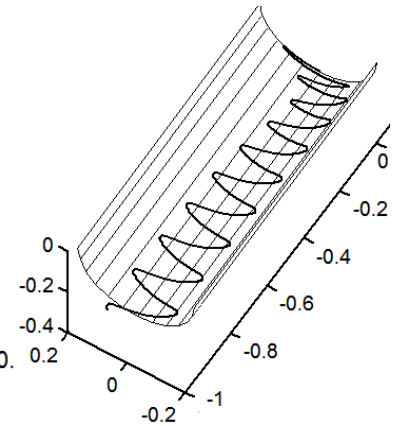

(b)

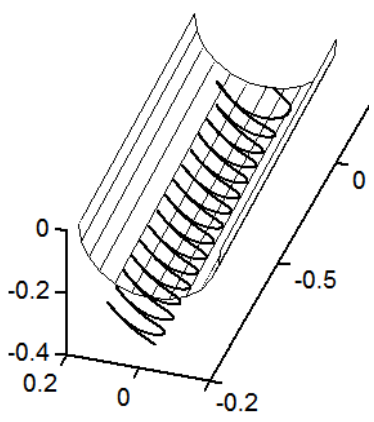

(c)

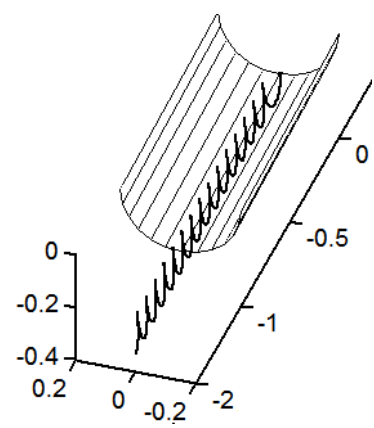

(d)

Fig. 4. Relative trajectories of particle sliding over an inclined cylinder $(\beta=29)$ :
(a) $\omega=10 \mathrm{~s}^{-1}, \varphi=90^{0}, t=10 \mathrm{~s}$;
(b) $\omega=20 s^{-1}, \varphi=90^{0}, t=3 s$;
(c) $\omega=10 s^{-1}, \varphi=45^{0}, t=10 s$;
(d) $\omega=10 s^{-1}, \varphi=15^{0}, t=10 \mathrm{~s}$. 
When comparing Figs. 4(a) and 4(b), it can be concluded that an increase in the frequency of oscillations results in an increase of the velocity of particle movement downwards over a cylinder. If a cylinder is oscillated not in transverse direction, but at a certain angle to it, the trajectory changes (Fig. 4 (c)(d)). Obviously, at $\varphi=0$ there are particle oscillations along a straight line - a lower generator of a cylinder.

Let us find out how a particle slides over a cylinder, which is inclined at a friction angle to a horizontal plane. If there are no oscillations after its motion is stabilized, a particle moves along a lower generator at stable speed. If a cylinder performs reciprocating rectilinear oscillations in transverse direction, the trajectory of particle motion is a space curve, which is similar to a sine curve with period that increases with time (Fig. 5(a)). A velocity curve (Fig. 5(b)) shows that it has a periodic nature, but, in general, it increases. Our investigations show that this increase along a cylinder axis has a linear character. A reaction $N$ curve shows that surface pressure of a particle at the highest points of the trajectory is close to zero.

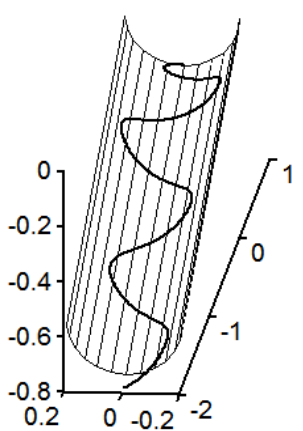

a)

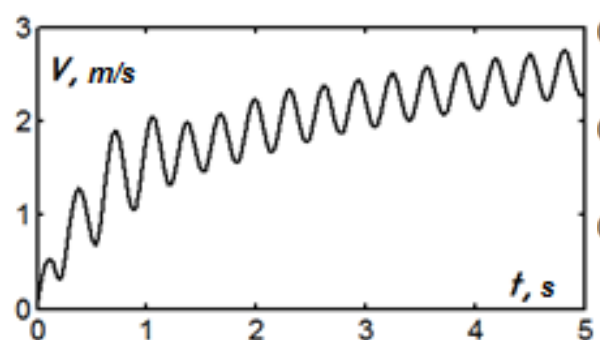

b)

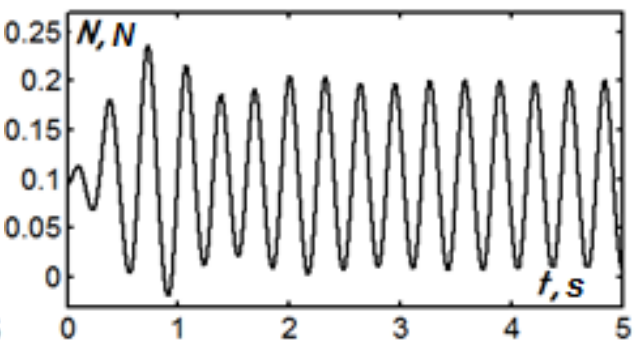

c)

Fig. 5. Graphic illustrations of particle motion over a cylinder, which is inclined at a friction angle $(\beta=\operatorname{arctg} \mathrm{f})$, that performs reciprocating oscillations:

(a) Trajectory of a particle, which begins its motion from the state of rest;

(b) Curves of surface velocity $\mathrm{V}$ of relative particle motion;

(c) Curves of surface reaction $\mathrm{N}$ of relative particle motion.

A cylinder performs reciprocating oscillation at an angle $\psi$ to a horizontal plane $(b=0, \psi \neq 0)$. Relative particle motion at transverse oscillations of a cylinder in a horizontal plane $\left(\varphi=90^{\circ}\right)$ is shown in Fig. 4(a). If angle $\varphi$ decreases, the distance, which is covered by a particle downwards along the axis, increases (Fig. 4(c)-(d)). Obviously, the covered distance is maximum at $\varphi=0$. Let us determine the influence of angle $\psi$ on the distance covered. For this purpose we take all the parameters of an oscillatory motion, for which the trajectory in Fig. 4(a) is plotted, and, in addition, we add certain values of angle $\psi$. If there is such oscillation, the height of cylinder points changes. Top view of Fig. 6(a) shows relative trajectories of particle motion at different values of angle $\psi$. At $\psi=90^{\circ}$ (that is to say, at vertical oscillations) a particle stops its motion at all. Figure 6(b) represents trajectories of particle sliding at angle $\varphi=45^{\circ}$ and various values of angle $\psi$ during time $t=7 \mathrm{~s}$. Unlike angle $\varphi$, angle $\psi$ influences particle movement in a different way. At its increase from zero, the speed of particle movement increases as well and it reaches the highest value at about $\psi=35^{\circ}$, and, afterwards, it begins to decrease. This is clear when considering the distance covered by a particle along the generators of a cylinder (Fig. 6(b)) at different values of angle $\psi$.

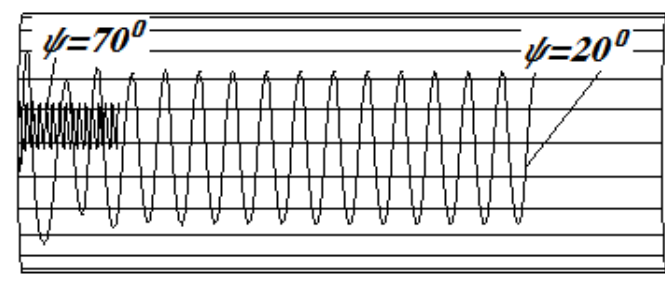

a)

Fig. 6. Relative trajectories of particle sliding over an inclined cylinder $\left(\beta=2^{0}\right)$ at various combinations of angles $\varphi$ and $\psi$ : (a) $\varphi=90^{\circ}, t=10 \mathrm{~s}$; (b) $\varphi=45^{\circ}, t=7 \mathrm{~s}$.

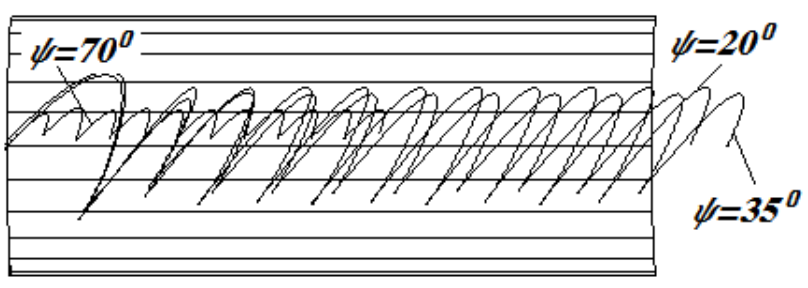

b) 
A general case of cylinder oscillation (all its points trace a curvilinear trajectory - a circle or an ellipse - in vertical parallel planes). If $a=b$, a circle is the trajectory of oscillations of cylinder points. Let us consider this case, as it is simple for technical implementation. Let us take an inclined cylinder with an inclination angle $\beta=2^{0}$, which is the same as in previous cases. Here, the value of angle $\psi$ does not make any difference. Fist of all, let us consider oscillations in the transverse direction relative to the cylinder (at $\left.\varphi=90^{\circ}\right)$. Figure $7(\mathrm{a})$ represents the trajectories of particle sliding at various values of angle $\varphi$ at $\beta=2^{0}, R=0.2 \mathrm{~m}, \omega=10 \mathrm{~s}^{-1}$, $a=b=0.1 \mathrm{~m}, a=\pi / 2, a^{\prime}=0, u^{\prime}=0, f=0.3, t=10 \mathrm{~s}$. After its motion is stabilized, a particle begins to trace a helical line on the surface moving downwards (that is to say, its motion over half of a cylinder is not possible). However, at $\varphi=45^{\circ}$ there is an unexpected effect: a particle begins to move upwards without getting outside a half cylinder. This effect is greater at $\varphi=15^{0}$.

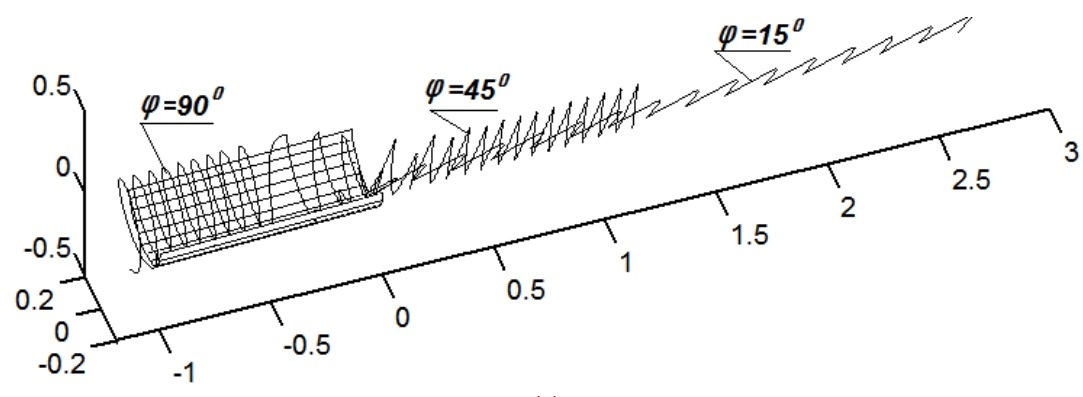

(a)

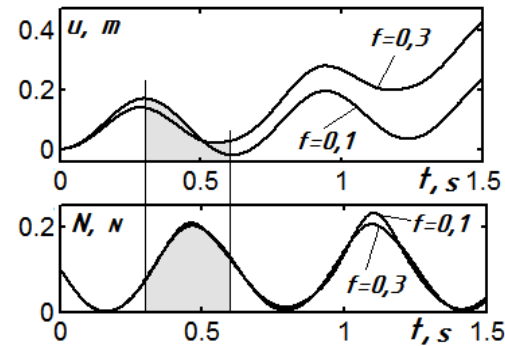

(b)

Fig. 7. Graphic illustrations of particle motion over a cylinder that is inclined at an angle $\beta=2^{0}$, the points of which trace circles:

(a) trajectories of a particle for various values of angle $\varphi$;

(b) curves of distance $u$ and surface reaction $N$ for $\varphi=15^{0}$.

In order to explain this effect, Fig. 7(b) presents a distance $u$ curve (at the top) and a surface reaction $N$ curve depending on various friction coefficients. Vertical lines display the curves part, when the distance $u$ decreases, that is to say, when a particle moves down. This happens when surface reaction is of the highest value, thus, friction force is of the highest value as well. Upward movement under inertia force happens, when friction force values are lower, that is to say, inertia force dominates over friction force and weight force component. Surface reaction insignificantly depends on friction coefficient and friction force directly depends on it. This explains the fact that, if friction coefficient decreases, a particle moves upwards more slowly. If $f=0$, it does not move upwards, it moves downwards. It can be concluded that, when moving upwards along a cylinder, a particle performs oscillatory motion, which is represented by the trajectory (Fig. $7($ a)). Obviously, at $\varphi=0$ this effect further increases, but is it not possible to see particle oscillations from its trajectory form, since it coincides with a lower rectilinear generator of a cylinder.

It can be assumed that if there is a certain relation of semi-axes $a$ and $b$, that is to say, when the points of a cylinder move around an ellipse, and there is a certain value of angle $\psi$, this effect can be increased. However, our investigations show that in order to move particles upwards, the best trajectory of relative motion of a cylinder is a circle. Here, the direction of angular velocity $\omega$ makes a difference. If $\omega$ takes a negative sign, which means a change in the direction of rotation of cylinder points around circles, at all the same previous parameters, particles move not upwards over a cylinder, but downwards, and, here, the velocity of their motion in greater than the one for upwards movement. Fig. 8, a represents trajectories of particle motion downwards over a cylinder at various values of angle $\varphi$ (top view). The parameters are the same as in the previous case, except for angular velocity $\omega=-10 \mathrm{~s}^{-1}$ and time of movement $t=3 \mathrm{~s}$. Figure 8(a) shows that the trajectory is displaced from the axis of symmetry of a cylinder (it is easy to see from the example of a trajectory for $\left.\varphi=15^{\circ}\right)$. Figure 8(b) represents the curve of relative velocity of a particle. At a certain time moment (when a particle is at its lowest position on a cylinder) its velocity is close to zero. 


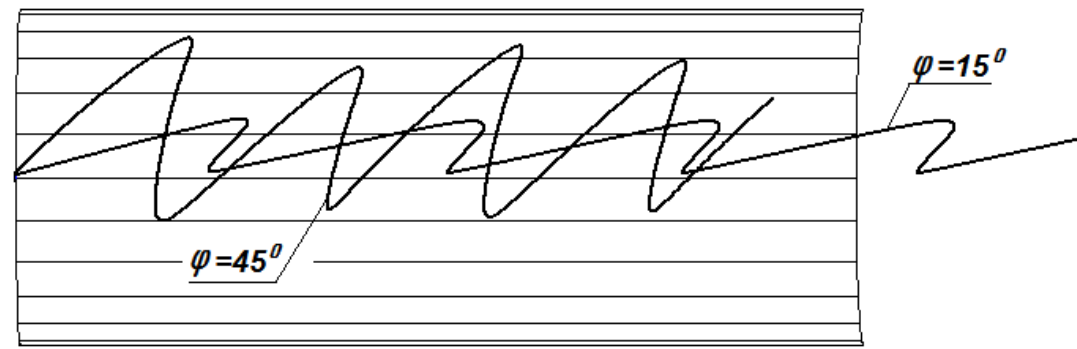

(a)

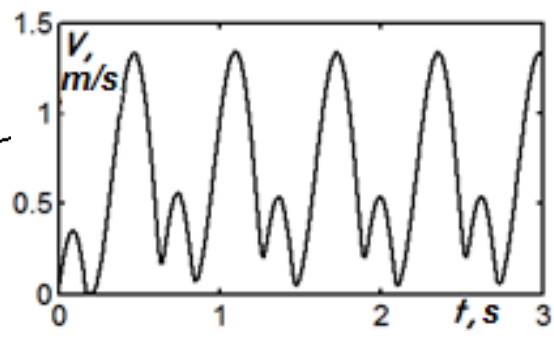

(b)

Fig. 8. Graphic illustrations of particle motion over a cylinder, the points of which move around a circle with angular velocity $\omega=-10 s^{-1}$ and which is inclined at an angle $\beta=2^{0}$ :

(a) Trajectories of a particle for various values of angle $\varphi$;

(b) Curve of relative velocity of a particle for $\varphi=15^{\circ}$.

\section{Conclusions}

The formulated differential equations provide means for solving the problems for determining kinematic parameters of particle motion over a cylinder, which performs translational oscillations in a vertical plane. A cylinder may be placed either vertically or at a preset angle to a horizontal plane. Trajectories for various cases of reciprocating oscillations of a cylinder in rectilinear direction have been traced. For reciprocating oscillations of an inclined cylinder, the points of which trace circles, there can be a case, when particles move upwards over a cylinder. The speed of upward movement depends on a friction coefficient. This condition can be used for practical purposes, when separating technological material by its friction performance. It is possible to choose such an angle of cylinder inclination, at which particles with a greater friction coefficient will move upwards and the ones with a lower friction coefficient will move downwards. When the direction of cylinder points rotation in circles changes, all the particles will move downwards, here, the speed of downward movement will be greater that the speed of upward movement.

\section{References}

[1] P. M. Vasylenko, Theory of Particle Motion over Rough Surfaces of Agricultural Machines. Kyiv, Ukraine: Ukrainian Academy of Agricultural Sciences, 1960.

[2] V. V. Gortinskii, A. B. Demskii, and M. A. Boriskin, Separation Process at Grain Processing Enterprises, 2nd ed. Moskov, Russia: Kolos, 1980.

[3] I. I. Blekhman and G. Yu. Dzhanelidze, Vibratory Displacement. Moskov, Russia: Nauka, 1964.

[4] I. I. Blekhman, Vibratory Mechanics. Moskow, Russia: Fizmatlit, 1994.

[5] P. M. Zaika, "Concerning one family of regular particle motion over an oscillating plane of vibratory grain-cleaning machine," Theory of Mechanisms and Machines, vol. 1. M. Gorkii Kharkiv State University, 1966, pp. 28-33.

[6] S. F. Pylypaka and M. B. Klendii, "Particle motion over the surface of a cylinder, all the points of which trace circles in horizontal planes," Bulletin of Sumy National Agrarian University Series Mechanization and Automation of Production Processes, vol. 10/3, no. 31, pp. 195-201, 2016.

[7] S. F. Pylypaka, M. B. Klendii, and O. M. Klendii, "Particle motion over the surface of a rotary vertical axis helicoid," INMATEH - Agricultural Engineering, vol. 51, no. 1, pp. 15-28, April 2017.

[8] P. Ratanavararaksa and M. Dejnakarintra, "Series solutions of the anharmonic motion equation $y^{\prime \prime}+y^{2}=$ c," Engineering Journal, vol. 20, no. 5, pp. 203-213, November 2016.

[9] R. B. Hevko, I. G. Tkachenko, S. V. Sinii, and I. V. Flonts, "Development of design and investigation of operation processes of small-sclale root crop and potato harvesters," INMATEH - Agricultural Engineering, vol. 49, no. 2, pp. 53-60, August 2016.

[10] R. B. Hevko, R. I. Rozum, and O. M. Klendii, "Development of design and investigation of operation processes of loading pipes of screw conveyors," INMATEH - Agricultural Engineering, vol. 50, no. 3, pp. 89-94, April 2016. 